Sylvia Mi chel ina Fernandes Brenna 1

Ellen Hardy 2

Luiz Carlos Zeferino 2

Iara Namura 1

\title{
Conhecimento, atitude e prática do exame de Papanicolaou em mulheres com câncer de colo uterino
}

\author{
Knowledge, attitudes, and practices \\ related to the Pap smear among women \\ with cervical cancer
}

1 Divisão Médica, Hospital-Maternidade Leonor Mendes de Barros, Secretaria de Estado da Saúde. Av. Cel so Garcia 2477, São Paulo, SP, 03015-000, Brasil. brenna.ops@zaz.com.br 2 Departamento de Tocoginecologia, Faculdade de Ciências Médicas, Universidade Estadual de Campinas. C. P. 6181, Campinas,SP 13081-970, Brasil.
Abstract Despite screening programs, Brazil has a high cervical cancer mortality rate. The objective of this cross-sectional study was to analyze knowledge, attitudes, and practices related to the Pap smear and to understand why women fail to submit to this screening test. A structured questionnaire was used to interview 138 women: 90 with high grade intraepi thelial neoplasia and 48 with invasi ve cervical cancer. Inadequate practices were more frequent among women with invasive cancer. In terms of difficulties in obtaining medical care,more than $80 \%$ of women reported lack of moti vation, $60 \%$ reported that physi cians failed to conduct a complete physical examination, and some 50\% reported that physi cians' schedul es were busy. Having a Pap smear usually depended on a physician's request and the woman being symptomatic. Women over than 56 years old showed more frequent inadequate knowledge, attitudes and practices. However, those with more schooling were more knowledgeable of the Pap smear procedure. Age and less schooling could be barriers against women participating in screening programs, but soci oeconomic problems must also be consi dered for improving practices related to the Pap smear.

Key words Uterine Neoplasias; Women's Health; Medical Examination

Resumo O câncer de colo uterino apresenta alta mortalidade no Brasil, apesar dos programas para rastreamento. O obj etivo deste estudo, de corte transversal, foi analisar conhecimento, atitude e prática do exame de Papanicolaou e entender a não adesão das mul heres a este exame. Foram entrevistadas 138 mulheres: noventa com neoplasia intra-epitelial de al to grau e 48 com câncer invasivo de colo uterino. As mulheres com câncer invasi vo tiveram prática mais inadequada do exame. No entanto, independente do diagnósti co, mais de $80 \%$ delas referi ram desmotivação/vergonha, $60 \%$ relataram que os médicos não exami navam e, cerca de $50 \%$ apontaram o tempo de espera para a consul ta e a demora no agendamento como dificuldades para serem atendidas. Em geral, a prática do exame dependeu da iniciativa do médico e a periodicidade da coleta foi determinada pel a procura de consulta devido a sintomas. As mul heres com 56 anos ou mais mostraram maior inadequação no conhecimento, na atitude e na prática. No entanto, aquel as com maior escolaridade conheciam melhor o exame. A maior idadee a menor escolaridade podem estar associados a não adesão das mulheres ao exame, porém as dificuldades sociais e econômicas para conseguir atendimento em saúde preci sam ser consi deradas para aumentar a prática do exame.

Palavras-chave Neoplasias Uterinas; Saúde da Mulher; Exames Médicos 
Introdução

O câncer de colo uterino ainda é um problema de saúde pública em países em desenvolvimento, pois apresenta altas taxas de prevalência e mortalidade em mulheres de nível social e econômico baixo e em fase produtiva de suas vidas. Estas mulheres, uma vez doentes, ocupam leitos hospitalares, o que compromete seus papéis no mercado de trabal ho e as priva do convívio familiar, acarretando um prejuízo social considerável.

Um marco histórico importante no conhecimento do câncer de colo uterino foi o estudo de Papanicolaou \& Traut (1943, apud Chong, 1990), que mostrou ser possível detectar células neoplásicas mediante o esfregaço vaginal. Assim, o exame de Papanicolaou passou a ser utilizado por diversos países para o rastreamento populacional, na deteç̧ão precoce do câncer de colo uterino. É fato que a lenta evolução deste câncer possibilita o seu diagnóstico na fase intra-epitelial (não invasiva) em mulheres assintomáticas, quando o tratamento é de baixo custo e tem elevado percentual de cura (Aquino et al., 1986).

Lamentavelmente, países com limitados recursos não têm expandido satisfatoriamente os serviços de rastreamento populacional para o câncer de colo uterino. Já foi proposto realizar exames citológicos com mai or intervalo entre os control es e nos grupos etários de maior risco, visando otimizar os recursos disponíveis (WHO, 1988). M étodos diagnósticos simplificados têm sido testados, como a inspeção visual do colo uterino, visando reduzir a dependência tecnológica (University of Zimbabwe/ Cervical Cancer Project,1999). Ainda que em fase de teste, a tipagem do Papiloma vírus humano (HPV) e a respectiva vacinação têm sido apontadas como possibilidades futuras ( Walboomers et al., 1999).

Todavia, grande parte destes estudos tem abordado a prevenção do câncer do colo uterino por um prisma eminentemente técnico, ao passo que as questões sociais, culturais e as características dos serviços de saúde não são consideradas, ou são, porém de forma muita simplista. As condições em que se têm realizado estes estudos não reproduzem as características gerais da população e dos serviços de saúde dos países em desenvolvimento.

No Brasil, segundo o Instituto Nacional do Câncer (INCA, 1999), o câncer de colo uterino representa a segunda causa de mortalidade bruta entre as neoplasias malignas para a população feminina nas regiões Sul, Sudeste, Nordeste e Centro-Oeste, ea primeira causa na Re- gião Norte. Estima-se que apenas $2 \%$ das muIheres em âmbito nacional, e cerca de $10 \%$ delas em grandes cidades como São Paulo e Rio de Janeiro, faziam o exame de Papanicolaou periodicamente na década de 70 (Aquino et al., 1986). É possível que no final da década de 80 , a prática do exame tenha chegado a $8 \%$ em nível de programa nacional, mas se sabe que esta se elevou a quase $30 \%$ no Município de São Paulo (Secretaria de Saúde de São Paulo, 1998).

Conseqüentemente, se a cobertura populacional do exame de Papanicolaou, no Brasil, foi baixa nas últimas décadas, não houve significativa redução da taxa de mortalidade por este câncer nos últimos anos. Por isso, este estudo teve como objetivo analisar conhecimento, atitude e prática e identificar fatores associados à baixa adesão de mulheres ao exame de Papanicolaou.

\section{Sujeitos e métodos}

Este estudo foi de corte transversal associado a inquérito CAP (Conhecimento, Atitude e Práti$\mathrm{ca}$ ), realizado com mulheres atendidas no Serviço de Oncologia Ginecológica do HospitalMaternidade Leon or Mendes de Barros. O hospital localiza-se na região leste do Município de São Paulo e é especializado em ginecologia e obstetrícia, atendendo a população usuária do sistema público de saúde. Os Centros de Saúde desta região encaminham para este Hospital as mulheres com exame de Papanicolaou anormal ou com manifestações clínicas de câncer do colo uterino.

A amostra foi cal culada com erro-a $5 \%$ (IC 95\%) e erro-b $20 \%$ (poder $80 \%$ ). Foram incluídas 138 mulheres atendidas consecutivamente, sendo 90 com o diagnóstico histológico de neoplasia intra-epitelial cervical (NIC) de alto grau e 48 mulheres com câncer invasivo do colo uterino. Foram excluídas as mulheres com menos de 20 anos e as com mais de 60, por elas não estarem incluídas no grupo etário considerado como população alvo para o rastreamento do câncer do colo uterino.

Cada mulher foi entrevistada uma única vez, durante a consulta médica no Hospital, utilizando um questionário estruturado na ocasião do diagnóstico. Todas as mulheres concordaram em participar espontaneamente, após leitura do Termo de Consentimento Informado. O conhecimento sobre o exame de Papanicolaou foi considerado adequado quando a mulher já tinha ouvido falar do mesmo e sabia que era para detectar câncer em geral ou especificamente de colo uterino; inadequado quan- 
do a mulher nunca tinha ouvido falar do exame ou já tinha ouvido, mas não sabia que era para detectar câncer. A atitude foi considerada adequada quando a mulher achou que fazer o exame era necessário; inadequada quando a mulher achou que fazer o exame era pouco necessário, desnecessário ou não tinha opinião sobre a sua necessidade. A prática foi considerada como adequada quando a mulher realizou seu último exame no máximo há três anos e, inadequada quando a mulher tinha feito o último exame há mais de três anos ou nunca o havia feito.

Foram variáveis independentes: idade para o diagnóstico; escolaridade; a mulher com trabal ho fora de casa. Para avaliar a causa da baixa adesão ao exame, também foram analisadas as dificuldades ou barreiras que as mulheres tiveram para procurar a consulta médica, os motivos que as levaram aos serviços de saúde e se a iniciativa de colher o exame de Papanicolaou foi delas ou dos médicos que as atenderam.

Os dados foram submetidos ao programa Statistical Analysis System (SAS Institute, 1990) e os resultados foram avaliados segundo a razão de chances - Odds Ratio (OR) ajustado por idade com IC 95\%. Para um melhor modelamento das variáveis, utilizou-se também a análise múltipla de regressão logística.

\section{Resultados}

Dentre as 138 mulheres entrevistadas, quase dois terços das mulheres com câncer in vasivo (63\%) tinham conhecimento inadequada do exame de Papanicolaou, a maioria (81\%) tinha atitude inadequada em relação à necessidade de fazê-lo e $56 \%$ praticavam-no de forma inadequada. Ainda, as mulheres com câncer invasivo mostraram chance 3,2 vezes maior (IC 95\%: 1,1-8,6) de praticar o exame inadequadamente com relação às mulheres com NIC, quando houve ajuste por idade. Com relação as mulheres com NIC, estes percentuais foram menores, apenas $41 \%$ não conheciam o exame ou a sua finalidade, $59 \%$ tin ham atitude desfavorável ou inadequada e $37 \%$ delas não o praticavam adequadamente (Tabela 1 ).

A mai oria das mulheres deste estudo, 79\% daquelas com NIC e 88\% das com câncer invasivo, consultava-se com o ginecologista apenas quando apresentavam sintomas. Durante as consultas ginecológicas, $82 \%$ das mulheres com NIC e 77\% das com câncer invasivo, relataram que era o médico quem tomava a iniciativa de colher o exame de Papanicolaou (Tabela 2).

As mulheres com 56 anos ou mais mostraram maiores chances de ter inadequação no co-
Tabela 1

Conhecimento, atitude e prática do exame de Papanicolaou segundo as mulheres com câncer de colo uterino.

\begin{tabular}{|c|c|c|c|c|}
\hline & Intra-epitelial (\%) & Invasivo (\%) & OR (IC 95\%) & OR* (IC 95\%) \\
\hline \multicolumn{5}{|l|}{ Conhecimento } \\
\hline Adequado & 59 & 37 & 1 & \\
\hline Inadequado & 41 & 63 & $2,3(1,1-5,2)$ & NS \\
\hline \multicolumn{5}{|l|}{ Atitude } \\
\hline Adequada & 41 & 19 & 1 & \\
\hline Inadequada & 59 & 81 & $3,0(1,2-7,6)$ & NS \\
\hline \multicolumn{5}{|l|}{ Prática } \\
\hline Adequada & 63 & 44 & 1 & 1 \\
\hline Inadequada & 37 & 56 & $2,2(1,0-4,8)$ & $3,2(1,1-8,6)$ \\
\hline Total (n) & 90 & 48 & & \\
\hline
\end{tabular}

* ajustado por idade

$\mathrm{NS}=$ não significante.

\section{Tabela 2}

Porcentagem de mulheres com câncer de colo uterino, com sintomas, para as quais o médico determinou a coleta para a realização do Papanicolaou.

\begin{tabular}{lcc}
\hline Variáveis & Intra-epitelial (n) & Invasivo (n) \\
\hline Consulta com sintomas & $79 \%(90)$ & $88 \%(48)$ \\
M édico pediu o exame & $82 \%(44)$ & $77 \%(13)$ \\
\hline
\end{tabular}

nhecimento com OR 8,6 (IC 95\%: 2,3-31,9), na atitude com OR 9,5 (IC 95\%: 1,1-80,4) e na prática com OR 4,1 (IC 95\%: 1,1-15,3), porém, aqueIas com maior escolaridade mostraram menor chance de ter conhecimento inadequado com OR 0,4 (IC 95\%: 0,1-0,9) (Tabela 3).

A desmotivação ou a vergonha foram as justificativas mais referidas para não fazer o exame, entre as mulheres com NIC e com câncer invasivo, $87 \%$ e $81 \%$ respectivamente. Com relação às dificuldades mais freqüentes geradas pelos serviços de saúde, foram referidas as seguintes: médicos que não as examinavam, relatado por $60 \%$ de todas as mulheres; tempo de espera longo para a consulta, relatado por $48 \%$ e $60 \%$ daquelas com NIC e câncer invasivo respectivamente; agendamento tardio, relatado por $47 \%$ das mulheres com NIC e $50 \%$ das com câncer invasivo (Tabela 4). 
Tabela 3

Fatores associados ao conhecimento, atitude e prática inadequados do exame de Papanicolaou em mulheres com câncer de colo uterino.

\begin{tabular}{lccc}
\hline Variáveis & Conhecimento & $\begin{array}{c}\text { OR*(IC 95\%) } \\
\text { Atitude }\end{array}$ & Prática \\
\hline $\begin{array}{l}\text { Faixa etária (anos) } \\
\text { Até } 40\end{array}$ & 1 & 1 & 1 \\
41 a 55 & $\mathrm{NS}$ & $\mathrm{NS}$ & $\mathrm{NS}$ \\
56 a 60 & $8,6(2,3-31,9)$ & $9,5(1,1-80,4)$ & $4,1(1,1-15,3)$ \\
Escolaridade (série) & & & - \\
Até 4 ạ \\
5a ou mais
\end{tabular}

* multi ajustado

NS = não significante.

Tabela 4

Porcentagem de mulheres com câncer de colo uterino segundo as dificuldades para consultar.

\begin{tabular}{llc}
\hline Dificuldades & Intra-epitelial & Invasivo \\
\hline Pessoais (n) & 63 & 43 \\
Desmotivação/vergonha & $87 \%$ & $81 \%$ \\
Distância & $19 \%$ & $40 \%$ \\
Filhos/parentes & $30 \%$ & $28 \%$ \\
Trabalho fora de casa & $29 \%$ & $28 \%$ \\
Dinheiro/transporte & $11 \%$ & $14 \%$ \\
Serviços de saúde (n) & 73 & 40 \\
Médico não examina & $60 \%$ & $60 \%$ \\
Tempo de espera & $48 \%$ & $60 \%$ \\
Agendamento tardio & $47 \%$ & $50 \%$ \\
Falta médico & $42 \%$ & $35 \%$ \\
Greve & $7 \%$ & $3 \%$ \\
\hline
\end{tabular}

\section{Discussão}

De acordo com os resultados deste estudo, as mulheres mais vel has tinham menor conhecimento sobre a finalidade do exame de Papanicolaou, tinham atitude mais desfavorável e não realizaram o exame nos últimos três anos. As mulheres com maior escolaridade tinham meIhor conhecimento sobre o exame. Observouse também que as mulheres com câncer invasivo tinham prática menos adequada do exame de Papanicolaou do que as mulheres com NIC.

A história das ações preventivas em câncer no Brasil é recente. As primeiras iniciativas pa- ra implantar a prevenção do câncer do colo uterino ocorreram no final da década de 60 , com progressos limitados ao longo da década de 70 (Zeferino et al., 1999). Em meados da década de 80, o Ministério da Saú de implementou o Programa de Atenção Integral à Saúde da Mulher (PAISM), em que um dos objetivos era aumentar a cobertura e a resolutividade dos serviços de saúde na execução das ações preventivas do câncer de colo uterino (Osis, 1998). Em seguida, começou a municipalização da saúde e implementação do Sistema único de Saúde (SUS) e recentemente, em 1997, foi instituído pelo INCA o projeto "Viva Mulher", que se tratava de um projeto piloto cujo objetivo era avaliar a baixa eficácia dos programas de prevenção existentes (INCA, 1997). Este último projeto propôs várias estratégias para estimular a adesão das mulheres à col eta do exame de Papanicolaou.

Sabe-se também que a cobertura populacional dos programas de controle de câncer do colo uterino é mais alta abaixo dos 40 anos (Nascimento et al., 1996). Usualmente, as muIheres mais jovens procuram mais os ginecologistas, possivelmente devido a eventos que são mais freqüentes neste grupo etário, tais como gravidez, necessidade de métodos anticoncepcionais ou tratamento de leu corréias. Parece que as mulheres com idades mais avançadas, especialmente depois da prole constituída e quando esterilizadas, procuram menos os serviços de saúde.

Sendo assim, é possível admitir que as muIheres mais vel has deste estudo são aquelas que nas décadas de 70 e 80 freqüentavam mais os serviços de saúde, quando se iniciava de forma ainda muito incipiente a realização do exame de Papanicolaou. Naquela época, falava-se muito pouco em prevenção de câncer e asações educativas e preventivas quase não existiam, de forma que seria esperado que as mulheres mais vel has tivessem um conhecimento inadequado do exame.

A deficiência do conhecimento do exame de Papanicolaou também é componente freqüente em mul heres mais velhas e com baixa escolaridade, em países em desenvolvimento, como mostraram estudos realizados em Santiago do Chile e Cidade do México (Alvarez, 1998; Aquilar et al., 1996). Em países desenvolvidos, as mulheres em geral sabem para que serve o exame de Papanicolaou e a deficiência de conhecimento é detectada apenas quando se pesquisam aspectos mais específicos sobre o câncer (Hasenyager, 1999; Pearlman et al., 1999). Todavia, minorias sociais em países desenvolvidos, como nos Estados Unidos, apre- 
sentam nível de conhecimento semelhantes às mulheres do presente estudo e também mostraram acesso limitado aos serviços de saúde (Kim et al., 1999; Risendal et al., 1999).

Outro resultado que é importante salientar é que muitas mulheres relataram dificuldades pessoais para procurar os serviços de saúde. A maioria das mulheres não tinha motivação, ou tinha vergonha, para procurar atendimento médico. Entretanto, problemas como distância, dificuldades para deixar filhos ou parentes, não poder deixar o trabal ho ou ainda ter dificuldades financeiras e com transportes também foram dificuldades apontadas.

Ainda, as mulheres citaram a má qualidade dos serviços de saúde como médicos que não examinam, tempo de espera para conseguir uma consulta, problemas com agendamento e consultas remarcadas por falta de médico ou greve. Tais dificuldades desesti mulam ou exigem que o tempo gasto para ter algum atendimento seja muito grande, levando as mulheres a deixarem seus afazeres diários para se ocuparem em conseguir atendimento médico. Esta situação poderia ser classificada como dificuldades pessoais das mulheres, mas, na realidade, seriam dificuldades geradas pelos próprios serviços de saúde.

Outro aspecto que este estudo mostra é que tanto as mulheres com NIC como aquelas com câncer invasivo, em geral, procuravam atendimento médico apenas quando tinham alguma queixa. A maioria dos atendimentos ginecológi cos é realizado devido a sintomas ginecológicos, anticoncepção, pré-natal e quando as muIheres levam os filhos ao atendimento pediátrico (Secretaria de Saúde de São Paulo, 1998). Uma vez que a mulher está presente no serviço de saúde, o exame de Papanicolaou é realizado por iniciativa do médico ou de outros profissionais de saúde. Deriva-se daí que a periodicidade da coleta do Papanicolaou é determinada pela presença de sintomas, que motivam a procura dos serviços de saúde.

No entanto, isto não significa que os profissionais de saúde estejam treinados e motivados para fazerem a coleta do Papanicolaou sis- tematicamente. Nesse caso, teremos uma situação de dupla passividade, em que a mulher não pede e ninguém Ihe oferece o exame. Além disso, o fato de as mulheres procurarem os serviços de saúde somente quando têm sintomas, seria uma característica sócio-econômica da população de países em desenvolvimento, devido ao entendimento de que não é necessário ir ao médico se não se sente nada.

Alguns países desenvolvidos somente conseguiram melhorar a cobertura populacional para o rastreamento do câncer de colo uterino quando introduziram uma sistemática de controlar e convocar as mulheres para fazer periodicamente o exame de Papanicolaou (Buehler \& Parsons, 1997; Elkind et al., 1990). No entanto, os países em desenvolvimento geralmente não têm um sistema de saúde organizado e, mesmo que o tivessem, há que se considerar problemática econômica da população em geral. Por exemplo, a falta de moradia própria e definitiva dificulta a convocação das mulheres para fazer exames periódicos. Assim seria pouco provável que um modelo organizacional de países desen volvidos fosse adequado também aos países em desenvolvimento.

Portanto, são muitas as dificuldades a serem vencidas a fim de aumentar a adesão das mulheres à col eta do exame de Papanicolaou. Este estudo apenas mostrou algumas características das mulheres que não fazem este exame e apontou algumas das deficiências dos serviços de saúde. Não é fácil modificar este cenário e a educação continuada para os profissionais de saúde bem como a educação em saúde para a população, realizadas isoladamente, tendem a ser quase inócuas. Também é um desafio às novas tecnologias, ainda que signifiquem maior simplicidade técnica. No Brasil, possivelmente, o modelo assistencial que foi construído ao longo das últimas décadas, baseado em especialidades, seja a grande dificuldade de base. É necessário construir um novo modelo que valorize as ações básicas de saúde e que promova uma interação mais compe tente com as características sociais e econômicas da população. 


\section{Referências}

AQUILAR, P. N.; PONCE, E. C. L.; RUIZ, P. A.; SANCHEZ, T. R.; URIZA, L. C. \& AVILA, M. H., 1996. Factors associated with Mexican women's familiarity with the purpose of the Pap test. Bulletin of the Pan American Heal th Organization, 30:348353.

AQUINO, E. M. L.; CARVALHO, A. I.; FAERSTEIN, E. \& RIBEIRO, D. C. S., 1986. Situação atual da detecção precoce do câncer cérvico-uterino no Brasil. Cadernos de Saúde Pública, 2:53-65.

ALVAREZ, S. L., 1998. Aspectos socio-culturales de la sexualidad como factores obstaculizantes de la prevención secundaria del cáncer cérvico uterino. Cadernos de Saúde Pública, 14(Sup. 1):33-40.

BUEHLER, S. K. \& PARSONS, W. L., 1997. Effectiveness of a call/ recall system in improving compliance with cervical cancer screening: A randomized controlled trial. Canadian Medical Association Journal, 157:521-526.

CHONG, C. Y. P., 1990. Estudo de Fatores Sócio-Demográfi cos Associados ao Conheci mento eà Realização do Exame de Papanicolaou. Dissertação de Mestrado, Campinas: Universi dade Estadual de Campinas.

ELKIND, A.; EARDLEY, A.; THOM PSON, R. \& SMITH, A., 1990. How district health authorities organize cervical screening. BMJ, 301:915-918.

HASENYAGER, C., 1999. Knowledge of cervical cancer screening among women attending a university heath center. Journal of the American College of Health, 47:221-224.

INCA (Instituto Nacional de Câncer), 1997. Viva MuIher - Programa Nacional de Controle do Câncer deColo Uterino. Normas e Manuais Técnicos. Rio de Janeiro: INCA.

INCA (Instituto Nacional do Câncer), 1999. Estimativa da Inci dência e Mortali dadepor Câncer. Normas e Manuais Técnicos. Rio de Janeiro: INCA.

KIM, K.; YU, E. S.; CHEN, E. H.; KIM, J.; KAUFMAN, M. \& PURKISS, J., 1999. Cervical cancer screening knowledge and practices among Korean American women. Cancer Nursing, 22:297-302.
NASCIMENTO, C. M. R.; ELUF NETO, J. \& REGO, R. A. A., 1996. Pap test coverage in São Paulo Municipality and characteristics of the women tested. Bulletin of the Pan American Health Organization, 30:303-311.

OSIS, M. J. M. D., 1998. PAISM: Um marco na abordagem da saúde reprodutiva no Brasil. Cadernos de SaúdePública, 14(Sup. 1):25-32.

PEARLMAN, D. N.; CLARK, M. A.; RAKOWSKI, W. \& EHRICH, B., 1999. Screening for breast and cervical cancer: The importance of knowledge and perceved cancer survivability. Women Health, 28: 93-112.

RISENDAL, B.; DEZAPIEN, J.; FOWLER, B.; PAPENFUSS, M. \& GIULIANO, A., 1999. Pap smear screening among urban Southwestern American Indian women. Preventive Medicine, 29:510-518.

SAS INSTITUTE, 1990. Statistical Analysis System. Cary: SAS Institute.

SECRETARIA DE SAÚ DE DE SÃO PAULO, 1998. Estratégia para Redução da Mortalidade por Câncer de Col o Uteri no no Estado de São Paulo. São Paulo: Secretaria de Saúde de São Paulo.

UNIVERSITY OF ZIMBABWE/CERVICAL CANCER PROJECT, 1999. Visual inspection with acetic acid for cervical cancer screening: Test qualities in primary care setting. Lancet, 353:869-873.

WALBOOMERS, J. M. M.; JACOBS, M. V.; MANOS, M. M.; BOSCH, F. X.; KUMMER, J. A.; SHAH, K. V.; SNIJDERS, P. J. F.; PETO, J.; MEIJER, C. J. L. M. \& MUNOZ, N., 1999. Human Papillomavirus is a necessary cause of invasive cervical cancer worldwide. Journal of Pathology, 189:12-19.

WHO (World Health Organization), 1988. Cytological Screningin the Control of Cervical Cancer. Geneva: WHO.

ZEFERINO, L. C.; COSTA, A. C.; MORELLI, M. G. L. D.; TAM BASCIA, J.; PANETTA, K \& PINOTTI, J. A., 1999. Programa de detecção do câncer do colo uterino de Campinas e região; 1968-1996. Revista Brasileira de Cancerologia, 45:25-33. 